\title{
萃取-表面增强拉曼光谱联用技术及其在有害物质 检测领域的应用
}

来永超 ${ }^{1}$, 陈静 $^{2}$, 占金华 ${ }^{2 *}$

1. 山东第一医科大学(山东省医学科学院), 医学科技创新中心, 济南 250062

2. 山东大学化学与化工学院, 胶体与界面教育部重点实验室, 济南 250100

*通讯作者, E-mail: jhzhan@sdu.edu.cn

收稿日期: 2021-02-01; 接受日期: 2021-05-07; 网络版发表日期: 2021-06-09 国家自然科学基金(编号: 82003503, 21575077, 2137068, 21075077)资助项目

摘要表面增强拉曼光谱(SERS)技术以其高灵敏度和分子特征指纹光谱在众多领域获得广泛应用，然而对于 实际复杂样品中的目标分析物，样品基质会极大地千扰目标分析物SERS信号的准确获取，从而限制SERS在实际 样品分析中的应用。萃取-表面增强拉曼光谱(Ex-SERS)联用技术为解决这一现实难题提供了可能性，国内外课题 组结合萃取和SERS的各自优势, 构建了基于固相萃取、固相微萃取、磁分散固相微萃取、薄层微萃取、液液分 散微萃取、擦拭萃取等多种Ex-SERS联用技术，并以此发展了面向多种有害物质的Ex-SERS联用方法，可实现复 杂基质中目标分析物的快速原位分离和SERS检测, 进一步拓展SERS在实际样品分析中的应用.

关键词表面增强拉曼光谱, 萃取, 样品前处理, 有害物质, 联用技术

\section{1 引言}

表面增强拉曼光谱(surface-enhanced Raman spectroscopy, SERS)作为一种强有力的原位化学分析技术, 不仅能提供目标分析物的特征指纹拉曼光谱以定性鉴 别被检测物, 还可以将被测分子的拉曼信号增强 6 个数 量级以上，从而实现被测分子的高灵敏检测. SERS现 已被广泛应用于环境分析、生化分析、食品分析和医 学诊断中 ${ }^{[1 \sim 5]}$.

\subsection{SERS在实际样品分析中存在的问题}

SERS增强机制主要为电磁增强和化学增强, 电磁
增强来源于入射电磁场与贵金属纳米结构(SERS基 底)内自由电子之间相互作用产生的表面等离子体共 振，电磁增强会随被检测分子与SERS基底间的距离增 大而呈指数衰减 ${ }^{[6,7]}$; 化学增强来源于被检测分子与 SERS基底之间的直接化学相互作用导致的被检测分 子拉曼散射截面的改变, 只发生于直接吸附在SERS基 底上的第一层分子 ${ }^{[8,9]}$. 由电磁增强和化学增强的原理 我们可以得出, 只有被检测分子距离SERS基底表面足 够近(通常 $2 \mathrm{~nm}$ 以内)时，才能获取有效的被检测物 SERS信号 ${ }^{[6-9]}$, 因此为进行高性能SERS 分析, 目标分 子必须能高效地吸附到SERS基底表面.

在通常的SERS分析中，会选用含有与SERS基底

引用格式: Lai Y, Chen J, Zhan J. Development of extraction-surface enhanced Raman spectroscopy and its application in detection of hazardous materials. Sci Sin Chim, 2021, 51: 665-678, doi: 10.1360/SSC-2021-0030 
高亲和力官能团(硫醇、羧酸、胺等)的被分析物，这 些被分析物很容易被吸附在SERS基底表面上, 从而获 得足够强的信号进行超灵敏SERS分析 ${ }^{[10,11]}$. 然而, 大 多数被分析物与SERS基底之间的亲和力较弱, 同时样 品基质中含有多种杂质, 杂质与目标分子在SERS基底 上会产生竞争吸附, 使被分析物无法接近基底表面, 降 低被分析物的信号强度并增加背景噪声水平, 甚至完 全得不到被分析物的信号 ${ }^{[12,13]}$. 这种竞争性吸附不仅 显著降低了SERS检测的敏感性和特异性, 而且还阻止 了SERS信号强度与被分析物浓度的相关性, 这就导致 常规SERS技术难以直接应用于复杂实际样品的分析 工作 ${ }^{[12,13]}$.

SERS分析中另一个普遍关心的问题是SERS的重 复性和定量性较差, 这通常是由于在SERS基底制备过 程中不可能保证每个区域都完全相同, 从而导致SERS 基底不同区域的增强性能和吸附性能有所差别, 进而 导致同一浓度的被分析物在不同SERS基底或者同一 SERS基底的不同区域产生的SERS信号强度有所差

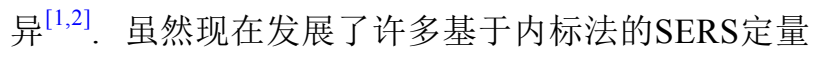
方法来消除SERS基底上不同区域SERS增强性能的差 异, 但是这些方法没有同时消除SERS基底上不同区域 对被分析物吸附性能的差异 ${ }^{[14]}$. 综上, 如何提高SERS 方法在实际样品分析中对被分析物的捕获灵敏度和专 一性以及重复性和定量性成为SERS领域的难点.

\section{2 萃取-表面增强拉曼光谱联用技术}

事实上不仅SERS技术，其他高灵敏的分析技术 (色谱、质谱、色质联用、电化学、苂光等)同样会受 到样品基质或杂质的强烈干扰, 因此在实际样品分析 工作中, 通常需要对样品进行必要的前处理. 萃取技 术是分析化学中最常用的样品采集、前处理和富集方 法, 可以将被分析物从复杂基质中高效地提取分离出 来, 用于进行下一步的分析 ${ }^{[15,16]}$. 萃取是通过被分析 物在两相(样品相与萃取相)中的分配系数不同而实现 分离富集目的, 从根本上来讲, 萃取的主要功能是分 离, 利用目标分析物与样品基质或杂质的物理化学性 质不同, 将一类物理化学性质相似的物质从组分复杂 的样品相中转移到组分简单的萃取相中, 然后经过脱 附/转移后用于进一步的分析 ${ }^{[15]}$. 需要注意的是, 萃取 通常不能实现单一被分析物的分离, 而是分离出一类 物理化学性质相似的物质.
萃取的效率通常取决于两相之间的总接触面积, 为了提高两相之间的总接触面积, 需要进一步压缩两 相的厚度, 基于此, 人们提出了固相萃取、固相微萃 取、磁分散固相微萃取多种萃取新技术 ${ }^{[17,18]}$, 尤其是 固相萃取技术, 将固定相(萃取功能层)压缩到接近单 分子层厚度(小于 $2 \mathrm{~nm})^{[18]}$, 这个厚度与SERS的高增强 区域不谋而合, 这就为萃取与SERS技术联用提供了技 术上的可能. 早在1999年, Carron课题组 ${ }^{[19]}$ 就用不同链 长的硫醇在SERS基底表面自组装形成疏水层, 并用于 捕获和SERS分析疏水性的被分析物, 这就在事实上实 现了SERS基底的萃取功能化, 形成了萃取与SERS联 用技术(Ex-SERS)的倠形.

将萃取技术与SERS进行联用, 有以下优势: 一方 面萃取相隔绝了被分析物与SERS基底的直接接触, 可 以有效避免样品基质和杂质对SERS 分析测定过程的 影响，同时避免了被分析物拉曼峰位置发生较大偏移 的化学增强过程的发生, 此外萃取相的存在赋予SERS 基底上不同区域对被分析物相同的吸附性能; 另一方 面被分析物总是与萃取相处于相同的SERS增强环境 中, 这样就可以利用萃取相作为内标来实现定量分析.

在Ex-SERS过程中, 由萃取的基本原理我们可以 得到在萃取平衡时有如下关系 ${ }^{[17]}$ :

$k=\frac{c_{2}}{c_{1}}$

其中, $k$ 为被分析物在两相中的分配系数, $c_{2}$ 为被分析 物在萃取相中的浓度, $c_{1}$ 为被分析物在样品相中的浓 度, 由于在Ex-SERS中萃取相的量远小于样品相的量, 所以在萃取前后样品中被分析物的浓度几乎不变, 由 此我们可以将上式写为

$k=\frac{c_{2}}{c_{1}} \approx \frac{c_{2}}{c_{0}}$

其中, $c_{0}$ 为被分析物在原始样品相中的浓度. 对于确定 的Ex-SERS体系中, $k$ 为固定值, 因此我们可以从上述 公式中得出, 在平衡状态下, 萃取相中被分析物的浓度 与原始样品中被分析物的浓度呈正比例关系.

如图1所示, 萃取技术与Ex-SERS联用技术具有很 多相同之处, 两者都具有萃取相和样品相, 有一些萃取 技术(如固相萃取、固相微萃取等)中包含支撑材料, 而在Ex-SERS中必须包含SERS活性基底, 因而从技术 上讲，包含支撑材料的萃取方法更容易与SERS技术进 行联用. 由于SERS增强能力会随着到基底表面距离的 

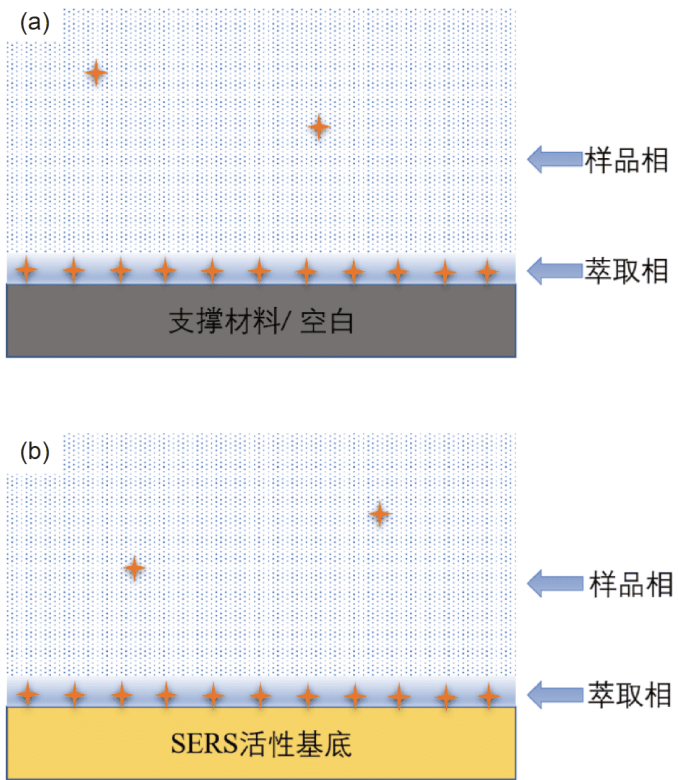

图 1 (a) 萃取技术示意图; (b) 萃取-表面增强拉曼光谱联用 技术示意图(网络版彩图)

Figure 1 (a) Illustration of extraction technology; (b) illustration of Ex-SERS technology (color online).

增大而急剧减小, 所以在Ex-SERS中萃取相要保持非 常薄(通常小于 $2 \mathrm{~nm}$ ) 以保证获得足够强的被分析物 SERS信号.

综上所述, Ex-SERS并不是单纯地将萃取与SERS 技术机械组合, 而是需要结合萃取和SERS的基本原理 和技术特点, 将萃取功能层修饰在SERS活性材料的表 面，并控制其厚度在SERS基底电磁增强有效的范围 内，实现被分析物在SERS基底表面的高效分离，进而 实现被分析物SERS定性和定量分析.

本文首先综述了用于Ex-SERS的基底制备与功能 化修饰技术和原理，介绍了国内外课题组基于固相萃 取、分子印迹固相萃取、固相微萃取、擦拭萃取、磁 分散固相微萃取、薄层微萃取、液液分散微萃取等开 发的Ex-SERS联用技术，并结合各自技术的特点讨论 了这些萃取技术与SERS联用的原理和技术，最后总结 了Ex-SERS联用技术的优缺点和发展方向.

\section{2 面向Ex-SERS应用的基底制备与萃取功 能化}

传统SERS基底的拉曼增强效果随时间快速衰减
且不能重复利用，同时SERS基底自身不具有萃取能 力, 因此无法直接利用原有的基底制备方法来构建ExSERS联用技术 ${ }^{[20]}$. 国内外课题组从提高可用于萃取功 能化SERS基底表面积和基底的可重复利用性、稳定 性等方面着手，发展了适用于Ex-SERS联用技术的 SERS基底制备方法. 同时，结合萃取技术的原理和理 论, 国内外课题组通过在上述SERS基底上修饰具有静 电相互作用、疏水相互作用的功能基团，建立了用于 Ex-SERS联用技术的SERS基底萃取功能化方法，为下 一步的Ex-SERS联用技术的开发与应用提供了可能.

\section{1 高稳定性Ex-SERS基底}

SERS基底中最常用的银纳米结构容易受到空气 和水的侵蚀，导致其SERS活性减弱，拉曼测试的聚焦 激光会导致很高的热积累，破坏基底上的贵金属纳米 结构, 进而导致 SERS 基底增强性能的不可逆损 伤 ${ }^{[20,21]}$. 这种SERS基底的时间不稳定性(尤其是在强 激光辐射下)可能导致光谱的多变和不可重复, 从而阻 碍SERS在环境分析中的进一步实际应用. 本课题组发 展了在商业铜箔上高可重复性和低成本性地制备可靠 SERS基底的湿化学方法, 制备了银纳米片覆盖的 SERS基底, 该基底不仅具有较高的信号增强效应和空 间均匀性，而且在强激光连续照射下仍可以保持极高 的时间稳定性(图2). X射线光电子能谱表明铜和银之 间因为费米能级差别而发生电子迁移，抑制了银纳米 结构的表面氧化, 另外铜和银两种金属极高的导热系 数也利于强激光下的基底散热, 我们由此提出了高稳 定SERS基底的制备策略: 保护表面原子不被氧化以保 持SERS基底的化学稳定性, 提高激光斑点处热量扩散 能力以维持SERS基底的物理稳定性，这种SERS基底 检测了4-巯基苯胺、甲拌磷、牛血清蛋白，结果表明 这种基底可以在强激光连续照射下保持稳定的SERS 增强效果，该研究为高稳定性表面增强拉曼光谱基底 的制备提供了一种新的思路 ${ }^{[20,22]}$.

多孔固体具有密度小、比表面积大、渗透性好等 优点，因此可广泛应用于制备各种固相萃取涂层材料. 本课题组采用电化学方法制备了具有SERS活性的多 孔银固相萃取丝, 具有纳米颗粒组成的堆集孔结构. EDS分析证明多孔纳米结构由 $\mathrm{Ag}$ 元素组成, 采用电化 学制备过程未造成多孔结构表面产生氧化银. 具有纳 米孔隙的银基微纳结构对萃取过程起着至关重要的作 
(a)

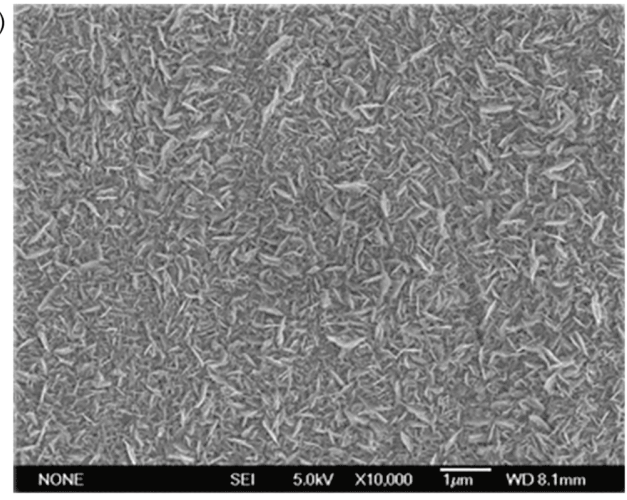

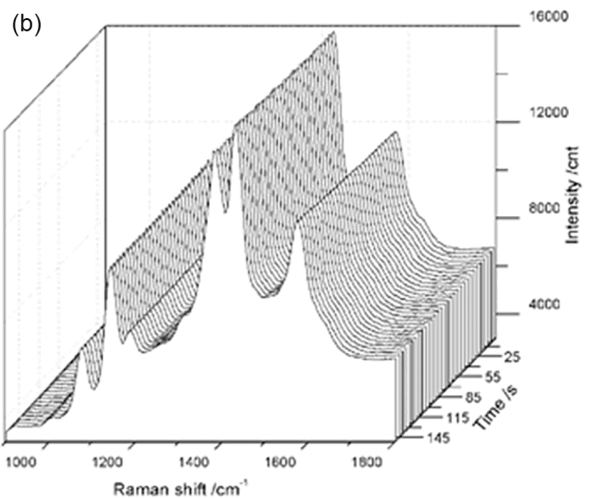

图 2 铜䈃上银纳米片组成的SERS基底(a)及其稳定性测试(b) ${ }^{[22]}$

Figure 2 SERS substrate constituted by silver nanoplates on copper foil (a) and its temporal stability (b) [22].

用，高比表面积可以提供更多可用于萃取功能化的位 点，该基底可作为固相萃取的骨架结构用于被分析物 的快速富集与检测 ${ }^{[23]}$.

在多孔银萃取丝的基础上, 我们通过多次循环伏 安法在银丝上制备多孔银, 然后利用卤素离子抑制 $\mathrm{AuCl}_{4}{ }^{-}$与 $\mathrm{Ag}$ 的置换反应, 在多孔银基底上沉积 $\mathrm{Au}$ 薄 膜，制备了一种超薄金层保护的多孔银纤维SERS基 底. 超薄金修饰层使该SPME-SERS纤维在加热下具有 强抗氧化性, 实验表明该纤维在 $200^{\circ} \mathrm{C}$ 加热下具有良好 的稳定性，并且在大气条件下具有良好的长期稳定性， 该基底的制备方法为高稳定性的SPME-SERS联用基 底的制备提供了可行的方案 ${ }^{[24]}$.

\section{2 自清洁型Ex-SERS基底}

氧化锌纳米棒作为一种典型的半导体材料, 具有 比表面积大、热稳定性好、易于制备等优点，因此氧 化锌纳米棒已经被广泛地用作固相萃取涂层. 形貌均 一的氧化锌纳米棒阵列, 同时也是理想的SERS基底支 撑介质 ${ }^{[25]}$. 本课题组利用晶种法在不锈钢丝表面原位 生长垂直的氧化锌纳米棒阵列, 然后室温下通过物理 离子溅射法在氧化锌表面溅射一层稠密的金纳米颗 粒, 生成具有SERS活性的三维结构的固相萃取骨架结 构(图3), 以此作为自清洁型的Ex-SERS纤维. 我们以4颈基苯胺为探针分子，在Ex-SERS纤维表面不同位置 测得的相对标准偏差为 $8.9 \%$, 表明该Ex-SERS纤维具 有良好的均匀性. 该Ex-SERS纤维用于检测结晶紫和 孔雀石绿, 线性范围分别为 $10^{-6} \sim 10^{-9} \mathrm{M}$ 和 $10^{-6} \sim 5 \times 10^{-9}$ $\mathrm{M}$ ，检测限分别达到 0.103 和 $0.241 \mathrm{nM}$. 该负载金-氧化
锌复合材料的不锈钢丝具有良好的光催化降解性能和 高拉曼增强能力, 可通过光催化降解清除表面检测物 质，实现基底的自清洁和循环使用. 实验表明该基底 可多次重复利用，同时保持拉曼增强能力基本不变， 该研究使表面增强拉曼光谱基底的可重复利用成为可 能 ${ }^{[26]}$

\section{3 面向擦拭萃取的柔性Ex-SERS基底}

本课题组通过溶剂热法制备了银纳米线溶胶，经 过过滤获得均匀的SERS活性萃取膜基底(图4), 该方 法制备的基底具有高时间稳定性和空间均匀性，这些 特性有助于减少光谱的漂移并节约整体分析时间. SERS活性萃取膜经特异性功能化修饰后可用于表面 擦拭萃取被测物, 实现多种多环芳烃、有机爆炸物、 无机爆炸物的富集和检测. 将银纳米线制备的柔性ExSERS基底擦拭萃取固体表面的目标分析物, 有利于减 少固体表面分析中样品采集和前处理的流程, 缩短了 分析时间, 极大地提高了分析效率 ${ }^{[27]}$.

\section{4 静电相互作用功能化Ex-SERS基底}

本课题组利用铜试剂-二乙基二硫代氨基甲酸钠 (DDTC)、颈基乙胺盐酸盐等实现了对Ex-SERS基底 的特异性修饰. DDTC通过 $\mathrm{Ag}-\mathrm{S}$ 键强相互作用修饰到 基底表面，从而生成带正电的SERS基底表面状态. 带 正电的银胶体保持固定大小和分散性. 带正电的银胶 体的Zeta电位约为 $32.1 \mathrm{mV}$, 而柠檬酸盐稳定的银胶体 的Zeta电位为 $-43.7 \mathrm{mV}$. X射线光电子能谱(XPS)和拉 曼光谱表明, 由于 DDTC 的双齿构型的吸附方式, 


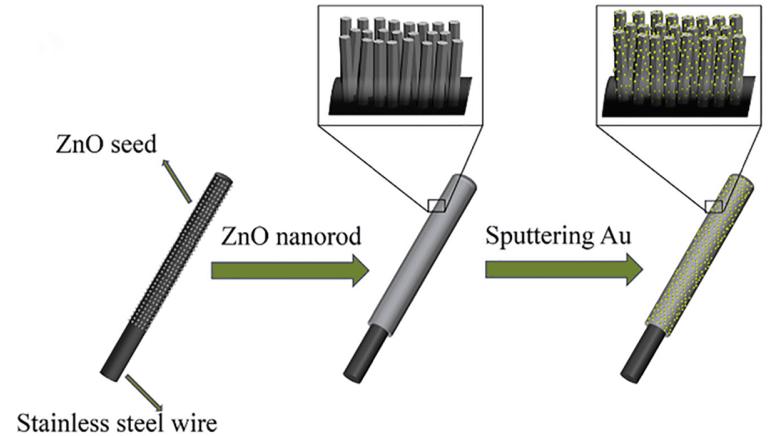

图 3 不锈钢纤维上 $\mathrm{Au}-\mathrm{ZnO}$ 纳米棒的制备过程 ${ }^{[26]}$ (网络版 彩图)

Figure 3 The fabrication process for the Au-ZnO NRs on the stainless-steel fiber [26] (color online).
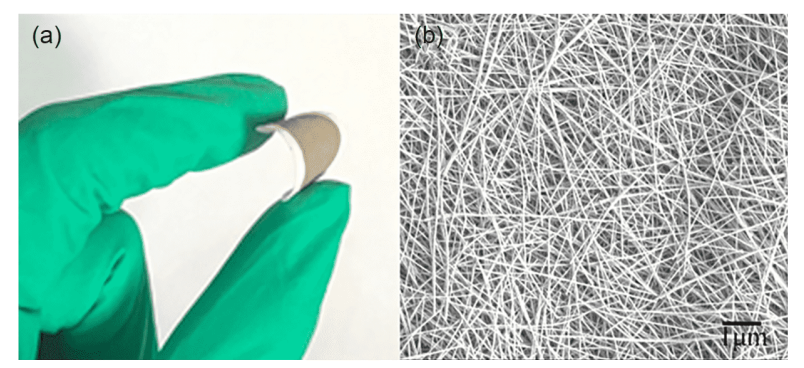

图 4 (a) 正电银纳米线膜基底的照片; (b) 银纳米线膜基底 的SEM照片 ${ }^{[27]}$ (网络版彩图)

Figure 4 (a) Photograph of the positively charged $\mathrm{Ag}$ nanowire membrane; (b) SEM image of the prepared membrane [27] (color online).

DDTC诱导了带正电的银纳米颗粒的形成, 从而导致 自由电子对在 $\mathrm{N}$ 原子中的重新分布以及 $\mathrm{C}=\mathrm{N}^{+}$键的形 成. 与伯胺或仲胺相比, DDTC作为改性剂即使在碱性 溶液中也可以保持胶体银的Zeta电位为正值. 这种带 正电的SERS基底可以利用静电相互作用, 通过擦拭萃 取捕获收集固体表面的待测物 ${ }^{[28]}$.

\section{5 疏水相互作用功能化Ex-SERS基底}

疏水性相互作用是萃取过程中最常用的目标分析 物捕获方式, 疏水相互作用虽然不具有特异性, 但是在 复杂基质的实际样品分析中，可以排除无机离子、极 性分子的干扰. 在固相萃取技术中，疏水相互作用通 过在固定相表面修饰疏水基团实现．在SERS基底上， 可以通过 $\mathrm{Au} / \mathrm{Ag}-\mathrm{S}$ 键强相互作用将烷基硫醇修饰到基 底表面形成疏水层. 多个课题组分别考察了不同链长 的烷基硫醇(丙硫醇、正己硫醇、1-十二硫醇)对SERS
信号增强能力的影响, 发现链长越短, 表面修饰对目标 分析物的SERS增强能力越大. 但是碳链越短, 对于被 分析物的富集能力越弱. 综合考虑, 大多数Ex-SERS分 析通常选取丙硫醇或正己硫醇作为SERS基底的疏水

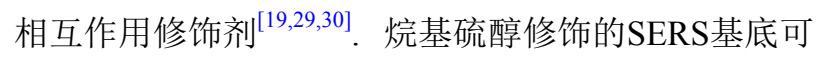
以作为一种通用的疏水性被测物的Ex-SERS平台.

\section{Ex-SERS联用方法的开发及其在有害物 质检测领域的应用}

针对Ex-SERS联用技术应用的空缺，国内外课题 组分别基于固相萃取、固相微萃取、分子印迹固相微 萃取、擦拭萃取、磁分散固相微萃取、薄层微萃取、 液液分散微萃取等技术建立了Ex-SERS技术, 并以此 发展了针对多环芳烃、农药、抗生素和危险爆炸物等 的Ex-SERS联用检测方法, 实现了多种有害物质的ExSERS联用方法的定性和定量分析(表1).

\section{1 固相萃取-表面增强拉曼光谱联用}

固相萃取(SPE) 是在液固色谱基础上发展起来的 样品前处理技术, 利用固相萃取填料对样品中的被分 析物或杂质的保留作用将其吸附，然后用少量溶剂洗 脱后进入下一步分析工作中, SPE具有分离快速、有 机溶剂用量少、操作简便等优点, 在SPE中常用的萃 取相通常是含有 $\mathrm{C} 18 、 \mathrm{C} 8$ 、氨基、氰基的填料 ${ }^{[17]}$. 值 得注意的是SPE使用高比表面积的微孔填料, 以保证 对目标物的近似全部保留, 然后通过少量淋洗液将其 从SPE填料上全部洗脱, SPE的吸附过程是一个非平衡 吸附过程 ${ }^{[17]}$, 会导致被分析物在SPE填料的不均匀分 布，基于SPE柱上的原位分析技术可能会产生定量 困难.

在SERS分析过程中, 只有激光斑点处的被分析物 能对最终信号有贡献, 如果在SPE-SERS中延用SPE常 用的高比表面填料堆叠的方式，会导致极大的浪费和 定量困难, 同时微孔级别金银纳米结构也不是很好的 SERS基底. 因此在SPE-SERS中, 通常采用适中比表面 积的多孔金银纳米结构作为骨架, 并限制整个萃取相 的量, 实现对被分析物的平衡吸附, 从而建立萃取相 中与样品相中被分析物浓度的相关性, 进而实现定量 分析.

本课题组通过组装银纳米枝晶的方式制备了固相 
表 1 Ex-SERS领域的主要工作总结 ${ }^{\mathrm{a})}$

Table 1 An overview on Ex-SERS analysis ${ }^{\text {a) }}$

\begin{tabular}{|c|c|c|c|c|c|c|}
\hline 方法 & SERS基底 & 萃取层 & 被分析物 & 线性范围 & 检测限 & 文献 \\
\hline SPME-SERS & 多孔Ag@Au & 多孔银 & $\begin{array}{c}\text { 呋喃西林 } \\
\text { 氨基䐖 }\end{array}$ & $\begin{array}{l}0.1 \sim 5.0 \mathrm{nM} \\
0.1 \sim 7.0 \mathrm{nM}\end{array}$ & $\begin{array}{l}5.1 \mathrm{ppb} \\
7.3 \mathrm{ppb}\end{array}$ & [24] \\
\hline SPME-SERS & $\mathrm{Ag}$ 纤维 & 多孔银 & $\begin{array}{l}\text { 三甲基氯化锡 } \\
\text { 三丁基氯化锡 } \\
\text { 三苯基氯化锡 }\end{array}$ & $\begin{array}{c}0.05 \sim 1.0 \mu \mathrm{M} \\
5 \sim 100 \mathrm{nM} \\
3 \sim 100 \mathrm{nM}\end{array}$ & $\begin{array}{l}6.9 \mathrm{ppb} \\
1.1 \mathrm{ppb} \\
0.2 \mathrm{ppb}\end{array}$ & {$[25]$} \\
\hline SPME-SERS & $\mathrm{Au}-\mathrm{ZnO}$ 纳米棒 & $\mathrm{Au}$ & $\begin{array}{l}\mathrm{CV} \\
\mathrm{MG}\end{array}$ & $\begin{array}{l}10^{-6} \sim 10^{-9} \mathrm{M} \\
10^{-6} \sim 10^{-9} \mathrm{M}\end{array}$ & $\begin{array}{l}0.103 \mathrm{nM} \\
0.241 \mathrm{nM}\end{array}$ & [26] \\
\hline swabbing- SERS & 银纳米线膜 & DDTC & $\begin{array}{l}\mathrm{ClO}_{4}^{-} \\
\mathrm{ClO}_{3}^{-} \\
\mathrm{NO}_{3}^{-}\end{array}$ & $\begin{array}{c}2.0 \sim 198.9 \mathrm{ng} \\
1.7 \sim 166.9 \mathrm{ng} \\
0.1 \sim 124 \mathrm{ng}\end{array}$ & $\begin{array}{l}0.43 \mathrm{ng} \\
1.46 \mathrm{ng} \\
0.09 \mathrm{ng}\end{array}$ & [27] \\
\hline Ex-SERS & Ag NPs & DDTC & $\begin{array}{l}\mathrm{ClO}_{4}^{-} \\
\mathrm{NO}_{3}^{-} \\
\text {苦味酸 }\end{array}$ & $1 \sim 500 \mathrm{ng} / \mathrm{cm}^{2}$ & $\begin{array}{l}1.19 \mathrm{ng} / \mathrm{cm}^{2} \\
2.01 \mathrm{ng} / \mathrm{cm}^{2} \\
8.96 \mathrm{ng} / \mathrm{cm}^{2}\end{array}$ & [28] \\
\hline Ex-SERS & Ag NPs 聚集体 & 正己硫醇 & PBDE 15 & $2.5 \times 10^{2} \sim 5.0 \times 10^{3} \mu \mathrm{g} / \mathrm{L}$ & $1.2 \times 10^{2} \mu \mathrm{g} / \mathrm{L}$ & [29] \\
\hline SPE-SERS & $\mathrm{Ag}$ 枝晶 & 丙硫醇 & 荧䓤 & $0.01 \sim 100 \mu \mathrm{g} / \mathrm{mL}$ & $0.01 \mu \mathrm{g} / \mathrm{mL}$ & [30] \\
\hline SPE-SERS & $\mathrm{Ag}$ 纳米片 $/ \mathrm{Cu}$ & $\mathrm{Ag}$ 纳米片 & 甲拌磷 & $3.84 \sim 288 \mathrm{nM}$ & $1.4 \mathrm{nM}$ & [31] \\
\hline MISPE-SERS & Ag NPs & MIPs & 噻苯咪唑 & $4 \sim 10 \mathrm{mg} / \mathrm{L}$ & $4 \mathrm{ppm}$ & [32] \\
\hline MISPE-SERS & Ag NPs & MIPs & $\begin{array}{l}\text { 咖啡因 } \\
\text { 甲拌磷 }\end{array}$ & $\begin{array}{c}0 \sim 100 \mu \mathrm{g} / \mathrm{L} \\
0 \sim 15 \mathrm{ppm}\end{array}$ & $\begin{array}{l}100 \mathrm{ng} / \mathrm{L} \\
0.02 \mathrm{ppm}\end{array}$ & [33] \\
\hline SPME-SERS & $\mathrm{Au}$ /不锈钢丝 & & $\begin{array}{c}\text { 水胺硫磷 } \\
\text { 福美铁 }\end{array}$ & $\begin{array}{l}0 \sim 15 \mathrm{ppm} \\
0 \sim 15 \mathrm{ppm}\end{array}$ & $\begin{array}{l}0.02 \mathrm{ppm} \\
0.05 \mathrm{ppm}\end{array}$ & [34] \\
\hline SPME-SERS & 多孔银纤维 & 半胱氨酸 & $\mathrm{PCP}$ & $0.01 \sim 7.0 \mu \mathrm{M}$ & $6.4 \times 10^{-9} \mathrm{M}$ & {$[35]$} \\
\hline SPME-SERS & Ag NPs & 石墨烯 & $\mathrm{BPA}$ & $2 \sim 100 \mu \mathrm{g} / \mathrm{L}$ & $1 \mu \mathrm{g} / \mathrm{L}$ & [36] \\
\hline SPME-SERS & $\mathrm{Ag}$ 包覆合金纤维 & rGO & $\begin{array}{l}\text { 磺胺嘧啶 } \\
\text { 磺胺甲恶唑 }\end{array}$ & $\begin{array}{l}0.01 \sim 100 \mu \mathrm{g} / \mathrm{cm}^{3} \\
0.01 \sim 100 \mu \mathrm{g} / \mathrm{cm}^{3}\end{array}$ & $\begin{array}{l}1.9 \mathrm{ng} / \mathrm{cm}^{3} \\
4.4 \mathrm{ng} / \mathrm{cm}^{3}\end{array}$ & [37] \\
\hline EX-SERS & $\mathrm{Ag} \mathrm{NPs} / \mathrm{Au}$ & $\mathrm{Ag}$ NPs & $\begin{array}{l}\text { 福美双 } \\
\text { 若葱 }\end{array}$ & $\begin{array}{c}2.4 \sim 240 \mathrm{ng} / \mathrm{cm}^{2} \\
40.45 \sim 28315 \mathrm{ng} / \mathrm{cm} 2\end{array}$ & $\begin{array}{c}0.2 \mathrm{ng} / \mathrm{cm}^{2} \\
40.45 \mathrm{ng} / \mathrm{cm}^{2}\end{array}$ & [38] \\
\hline swabbing- SERS & Ag NWs & 丙硫醇 & $\begin{array}{l}\text { 葱 } \\
\text { 芘 }\end{array}$ & $\begin{array}{l}40.45 \sim 28315 \mathrm{ng} / \mathrm{cm} 2 \\
40.45 \sim 28315 \mathrm{ng} / \mathrm{cm} 2\end{array}$ & $\begin{array}{l}35.65 \mathrm{ng} / \mathrm{cm}^{2} \\
45.45 \mathrm{ng} / \mathrm{cm}^{2}\end{array}$ & [39] \\
\hline MSPME-SERS & $\mathrm{Fe}_{3} \mathrm{O}_{4} @ \mathrm{Ag}$ & & 砷 & $10 \sim 1000 \mu \mathrm{g} / \mathrm{L}$ & $10 \mu \mathrm{g} / \mathrm{L}$ & [40] \\
\hline MSPME-SERS & $\mathrm{Fe}_{3} \mathrm{O}_{4} @ \mathrm{GO} @ \mathrm{Ag}$ & $\mathrm{GO}$ & $\begin{array}{c}\text { 福美双 } \\
\text { 噻苯咪唑 }\end{array}$ & $\begin{array}{c}0.48 \sim 48000 \mathrm{ng} / \mathrm{cm}^{2} \\
4 \sim 40000 \mathrm{ng} / \mathrm{cm}^{2}\end{array}$ & $\begin{array}{c}0.48 \mathrm{ng} / \mathrm{cm}^{2} \\
40 \mathrm{ng} / \mathrm{cm}^{2}\end{array}$ & [41] \\
\hline MSPME-SERS & $\mathrm{Fe}_{3} \mathrm{O}_{4} @ \mathrm{GO} @ \mathrm{Ag}$ & GO & 氯霉素 & $1.0 \times 10^{-8} \sim 1.0 \times 10^{-5} \mathrm{M}$ & $1.0 \times 10^{-10} \mathrm{M}$ & [42] \\
\hline MSPME-SERS & $\mathrm{Fe}_{3} \mathrm{O}_{4} @ \mathrm{SiO}_{2} @ \mathrm{Ag}$ & $\mathrm{SiO}_{2}$ & 枸椓酸西地那非 & $1.0 \times 10^{-9} \sim 1.0 \times 10^{-3} \mathrm{M}$ & $1.0 \times 10^{-8} \mathrm{M}$ & [43] \\
\hline MSPME-SERS & $\mathrm{CoFe}_{2} \mathrm{O}_{4} @ \mathrm{Au}$ & & $\begin{array}{c}\text { 4,4-硫代联苯胺 } \\
\text { 呋喃妥因 }\end{array}$ & $\begin{array}{c}0.054 \sim 21.7 \mathrm{mg} / \mathrm{L} \\
0.05 \sim 1.0 \mathrm{mg} / \mathrm{L}\end{array}$ & $\begin{array}{l}0.026 \mathrm{mg} / \mathrm{L} \\
0.014 \mathrm{mg} / \mathrm{L}\end{array}$ & [44] \\
\hline TFME-SERS & Au NPs & $\mathrm{ZnO}$ & $\begin{array}{c}\mathrm{SO}_{2} \\
\mathrm{SO}_{3}{ }^{2-}\end{array}$ & $\begin{array}{c}1 \sim 200 \mu \mathrm{g} / \mathrm{mL} \\
25 \sim 400 \mathrm{mg} / \mathrm{kg}\end{array}$ & $\begin{array}{c}0.1 \mu \mathrm{g} / \mathrm{mL} \\
6 \mathrm{mg} / \mathrm{kg}\end{array}$ & $\begin{array}{l}{[45]} \\
{[46]}\end{array}$ \\
\hline TFME-SERS & Au NPs & SWCNTs & 西草净 & $10 \sim 300 \mu \mathrm{g} / \mathrm{L}$ & $2 \mu \mathrm{g} / \mathrm{L}$ & [47] \\
\hline TFME-SERS & Au NPs & 硅胶板 & 苯甲酸钠 & $25 \sim 500 \mu \mathrm{g} / \mathrm{mL}$ & $3.6 \mu \mathrm{g} / \mathrm{mL}$ & [48] \\
\hline Ex-SERS & Ag NPs & 丙硫醇 & 苂葱 & $0.789 \sim 158 \mathrm{ng} / \mathrm{cm}^{2}$ & $0.27 \mathrm{ng} / \mathrm{cm}^{2}$ & [49] \\
\hline DLLME-SERS & Ag NPs & 丙硫醇 & 芘 & $11.9 \sim 395 \mu \mathrm{g} / \mathrm{L}$ & $0.50 \mu \mathrm{g} / \mathrm{L}$ & {$[50]$} \\
\hline Ex-SERS & $\mathrm{Ag} @ \mathrm{WS}_{2}$ & $\mathrm{WS}_{2}$ QDs & 福美双 & $12 \sim 1200 \mathrm{ppb}$ & $1.6 \mathrm{ppb}$ & [51] \\
\hline Ex-SERS & $\mathrm{Ag}$ 纳米片 $/ \mathrm{Cu}$ & 硫辛酸 & 苂蒽 & $0.1 \sim 10^{4} \mathrm{ng} / \mathrm{mL}$ & $6.73 \mathrm{pg} / \mathrm{mL}$ & {$[52]$} \\
\hline SPME-SERS & $\mathrm{Ag} / \mathrm{Cu}$ 纤维 & DDTC & $\mathrm{ClO}_{4}^{-}$ & $0.1 \sim 100 \mu \mathrm{M}$ & $6.0 \mathrm{ppb}$ & [53] \\
\hline SPME-SERS & $\mathrm{Ag} \mathrm{NPs} / \mathrm{Ag}-\mathrm{Cu}$ 纤维 & 丙硫醇 & 苂葸 & $10^{-6} \sim 10^{-8} \mathrm{M}$ & $7.56 \times 10^{-10} \mathrm{M}$ & [54] \\
\hline
\end{tabular}

a) SERS: 表面增强拉曼光谱; Ex: 萃取, SPME: 固相微萃取; SPE: 固相萃取; DLLME: 液液分散微萃取; swabbing: 擦拭萃取; MSPME: 磁 固相微萃取; TFME: 薄层微萃取; MIPs: 分子印迹聚合物; Ag NPs: 银纳米颗粒; Ag NWs: 银纳米线; QDs: 量子点; RGO: 还原氧化石墨烯; SWCNTs:单壁碳纳米管; DDTC: 铜试剂; PCP: 五氯酚; MG: 孔雀石绿; CV: 结晶紫; PBDEs: 多溴联苯醚; BPA: 双酚A 


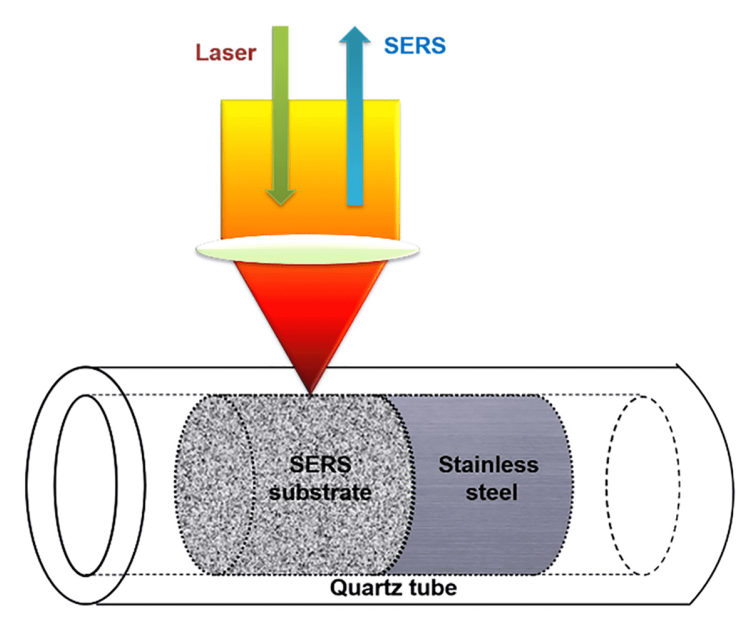

图 5 SPE-SERS联用柱示意图与SERS检测方式 ${ }^{[30]}$ (网络版 彩图)

Figure 5 Illustration of the structure of the SERS-active extraction column and the SERS detection mode based on the column [30] (color online).

萃取-表面增强拉曼光谱(SPE-SERS)联用分析柱(图5), 这种联用分析柱结合了固相萃取和表面增强拉曼光谱 技术特点, 不仅具有高度的时间稳定性(在连续高强度 激光照射下), 而且具有良好的可重复性(多次重复萃 取和洗脱). 利用这种联用分析柱, 可实现 $0.01 \sim 100 \mu \mathrm{g} / \mathrm{mL}$ 范围荧葱的快速定量检测, 对于单个荧葱样品, 整个分 析过程包括萃取、光谱获取、洗脱以及必要的中间连 接过程只需要不到 $30 \mathrm{~s}$, 这种面向多环芳烃快速分析 的SPE-SERS联用分析方法极大地简化了操作步骤, 节 约了总体分析时间 ${ }^{[20,30]}$.

本课题组利用改进的置换反应在铜网上原位生长 银纳米片, 获得银纳米片修饰的铜萃取膜. 银纳米片修 饰的铜萃取膜同时具有 SPE和SERS活性, 作为SPESERS基底能快速富集分析物。该SPE-SERS基底在循 环 5 次后，探针分子的相对标准偏差仅为 $12.13 \%$. 与传 统的滴滤SERS检测相比, 流动的SPE-SERS的信号强 度是滴滤法的 28 倍. 我们利用该基底对甲拌磷进行了 定性和定量分析. 在浓度范围为3.8 288 nM时，甲拌 磷特征拉曼峰强度与浓度呈良好的线性关系 $\left(R^{2}=0.98\right)$, 最低检出限 (LOD)为 $1.4 \mathrm{nM}(\mathrm{S} / \mathrm{N}=3)$. 利用该 SPE-SERS方法对实际水中的甲拌磷进行了定量检测, 证实了该方法在实际环境中对有机污染物的快速检测 有很大的实际应用价值 ${ }^{[31]}$.

本课题组利用正己硫醇修饰的银纳米颗粒聚集
体，基于SERS分析方法，实现了多环芳烃(PAHs)、多 溴联苯醚(PBDEs)的定性和定量分析. 我们利用该 SERS方法分析鉴别了PBDEs同分异构体，表明SERS 可以实现复杂基质中PBDEs的检测. 将硫醇修饰的基 底浸泡在BDE-3和BDE-15混合物溶液中, 对二者进行 SERS 分析. 尽管BDE-3和BDE-15的分子结构非常类 似, 仅有一个溴原子之差, 它们的SERS光谱也非常相 似. 由于SERS可以提供目标分析物的指纹特征光谱, 从而实现对PBDEs同分异构体的区分 ${ }^{[29,55]}$.

\section{2 分子印迹固相萃取-SERS联用技术}

常用的SPE技术通过单一作用力的方式对样品进 行前处理, 通常得到的是一类物理化学性质相似的物 质, 而不是单一的被分析物. 模拟酶-底物相互作用过 程诞生的分子印迹技术，利用分子印迹聚合物对印迹 分子进行多位点识别, 可以对印迹分子进行专一识别, 因此基于分子印迹技术的分子印迹固相萃取(MISPE) 被认为可以实现特异性分离被分析物. Lu课题组 ${ }^{[32]}$ 开 发了分子印迹固相萃取-SERS 联用方法(MISPESERS), 该方法通过合成的分子印迹聚合物可快速、 准确、灵敏地从复杂的橙汁中识别和提取噻苯达唑, 整个分析过程仅需 $23 \mathrm{~min}$, 噻苯达唑的检测限为 $4 \mathrm{ppm}$. 陆峰课题组 ${ }^{[33]}$ 利用负载银纳米颗粒的分子印迹聚合 物(MIP)开发了检测咖啡因的MISPE-SERS方法, 该方 法具有良好的均匀性(相对标准偏差, $\mathrm{RSD}=4.8 \%$ )和良 好的重复性 $(\mathrm{RSD}=8.7 \%$ ), 总分析时间为 $23 \mathrm{~min}$, 检出限 为 $100 \mathrm{ng} / \mathrm{L}$, 低于许多河流报道的咖啡因含量, 可用于 快速䇥选废水或其他水域中的咖啡因. 值得注意的是, 分子印迹中用于形成结合位点的聚合物通常具有很强 的拉曼散射截面, 这些聚合物会在最终的SERS谱图中 产生极高的背景信号, 可能干扰被分析物的定性分析, 并对被分析物定量检测下限产生不利影响.

\section{3 固相微萃取-表面增强拉曼光谱联用}

固相微萃取(SPME)是在气液/液液色谱基础上发 展起来的一种样品前处理技术, 以纤维材料作为基体, 在其表面涂渍高分子薄层形成萃取固定相, 通过“相似 相溶”的原理实现待测物的分离、富集和解吸 ${ }^{[18]}$. 与 SPE不同, SPME萃取过程是一个平衡吸附过程，被分 析物在整个SPME纤维的活性区域内均匀分布, 可以 建立萃取相中与样品相中被分析物浓度的相关性 ${ }^{[18]}$, 
因此在固相微萃取-表面增强拉曼光谱联用(SPMESERS)中, 非常有利于建立定量分析关系. 为保证萃取 得到的被分析物总量能满足下一步分析的需求, SPME纤维中固定相的厚度通常需要达到几十微米, 而SERS 需要被分析物足够接近SERS基底表面, 因此 在SPME-SERS中, 萃取相的厚度通常被限制在 $2 \mathrm{~nm}$ 以下.

He课题组 ${ }^{[34]}$ 采用顶空固相微萃取(HS-SPME)与 SERS相结合的方法, 建立了一种简便、快速的气相有 机磷杀虫剂和有机硫杀菌剂的检测方法. 以不锈钢丝 为基体, 分别通过自组装和刻蚀法制备涂覆金纳米粒 子的SPME-SERS纤维. 结果表明使用刻蚀法制备的 SPME-SERS纤维具有更好的分析能力, 其对甲拌磷、 水胺硫磷和福美铁的检出限分别低至 $0.02 、 0.02$ 和 $0.05 \mathrm{ppm}$, 这种方法可以简单地扩展到广泛的挥发性 有机化合物的检测. 因为不需要热解吸过程, 相比气 相色谱(GC)方法, HS-SPME在保持相似的灵敏度下, 其分析过程更加快速、简便, 可以在某些情况下替代 气相色谱法来研究挥发性化学物质的潜力.

本课题组利用一种结合SERS和SPME的快速篮选 有机锡(OTCs)的方法, 原位合成了一层多孔银膜作为 SERS活性SPME吸附涂层, 研究了三甲基氯化锡、三 丁基氯化锡和三苯基氯化锡在该SPME-SERS基底上 的SERS光谱, 分别选取 554、1410和 $999 \mathrm{~cm}^{-1}$ 处的特 征峰用于定量分析, 并对提取条件进行优化. 多孔银 纤维良好的稳定性和均匀性保证了定量检测信号的准 确性，对于三甲基氯化锡、三丁基氯化锡和三苯基氯 化锡, 增强因子分别为 $1.36 \times 10^{5} 、 9.0 \times 10^{5}$ 和 $9.1 \times 10^{5}$, 最低检出限分别为 $6.9 、 1.1$ 和 $0.2 \mathrm{ppb}$. 该SPME-SERS 方法结合了预浓缩和高灵敏度的优点, 可促进OTCs在 进出口贸易消费品安全中的快速篮选 ${ }^{[25]}$.
本课题组还开发了一种静电相互作用驱动的 SERS-SPME联用方法用于快速检测环境水样中的五 氯酚. 在纳米多孔银纤维表面修饰半胱氨酸, 在纤维表 面形成正固定相. 使用浸入式SPME模式提取五氯酚, 并利用其338、378和 $480 \mathrm{~cm}^{-1}$ 处的特征谱带定量检测 五氯酚, 该方法可实现 $3.7 \times 10^{5}$ 的高增强因子和 $6.4 \times 10^{-9} \mathrm{M}$ 的低LOD. 该SPME-SERS方法为现场快速 检测环境介质中的离子或极性有机污染物提供了通用 的策略 ${ }^{[35]}$.

吴莉课题组 ${ }^{[36]}$ 通过在硅纤维修饰单层石墨烯包 覆的银纳米颗粒制备了一种SERS活性的SPME纤维, 并用于建立SPME-SERS联用方法检测水样中的微量 双酚A (BPA), 该方法具有极高的灵敏度和极佳的稳定 性, 对BPA的检测限可低至 $1 \mu \mathrm{g} / \mathrm{L}$, 均匀性和重现性的 相对标准偏差(RSD)分别为 $14 \%$ 和 $13 \%$. 水样中双酚A 的测定加标回收率为 $97 \% \sim 110 \%$. 该SPME-SERS方法 集被分析物的分离、富集和检测于一体，具有灵敏、 快速、简单等特点, 被认为在BPA环境分析中具有广 阔的应用前景.

本课题组通过在银-铜合金纤维上同时沉积由还 原氧化石墨烯和银组成的杂化物, 制备了同时具有 SPME和SERS功能的针状涂层合金纤维. 该纤维是一 种可行的工具, 用于非破坏性采样和SERS检测组织模 拟物中抗生素磺胺嘧啶和磺胺甲恶唑的含量(图6). 我 们选择磺胺嘧啶 $1149 \mathrm{~cm}^{-1}$ 和磺胺甲恶唑 $1144 \mathrm{~cm}^{-1}$ 的 SERS峰作为定量分析的参考峰, 该方法的线性范围为 $0.01 \sim 100 \mu \mathrm{g} / \mathrm{cm}^{3}$, 磺胺嘧啶的检出限为 $1.9 \mathrm{ng} / \mathrm{cm}^{3}$, 磺 胺甲恶坐的检出限为 $4.4 \mathrm{ng} / \mathrm{cm}^{3}$. 该方法作为一种非破 坏性的分析方法, 可以有效缩短组织样品的提取和前 处理时间，同时该方法的检出限远低于欧盟所采用的 磺胺类抗生素的最大残留量, 表明该SPME-SERS方法
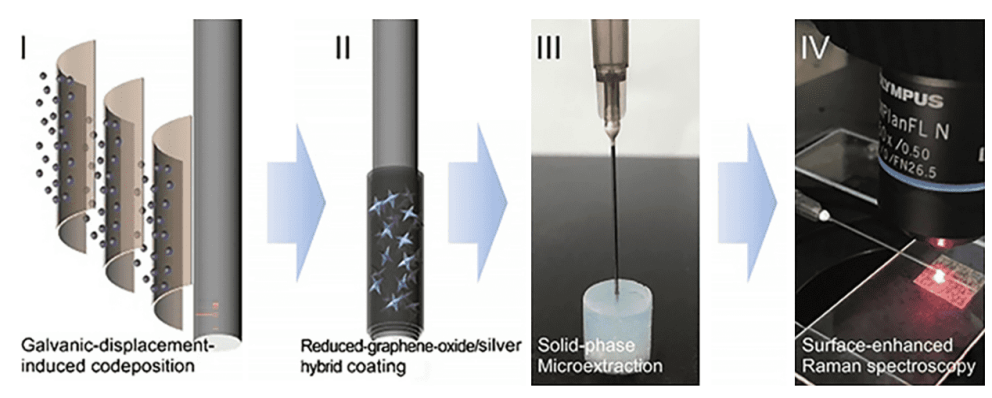

图 6 还原氧化石墨烯和银共沉积纤维用于SPME-SERS 非破坏性检测组织中的抗生素过程示意图 ${ }^{[37]}$ (网络版彩图)

Figure 6 Process of non-destructive SPME@SERS analysis of antibiotics with reduced-graphene-oxide/silver on alloy fibers [37] (color online). 
是一种很有前景的抗生素分析方法 ${ }^{[37]}$.

本课题组利用超薄金保护的多孔银纤维实现呋喃 酮和氨基嫝的SPME提取和SERS检测，考察了呋喃酮 和氨基脲的萃取和 SERS 性能, 分别选择 1350 和 $1387 \mathrm{~cm}^{-1}$ 的谱带作为特征峰进行定量测定，对方法进 行优化后，可实现呋喃酮和氨基嫝的定量检测，呋喃酮 和氨基嫝的LOD分别为5.1和7.3 ppb. 同时该SPME-SERS 纤维在加热下具有强抗氧化性, 并且在大气条件下具有 良好的耐久性. 这项工作为快速检测复杂基质中的禁用 抗生素及其标记残留物提供了一种可靠的方法 ${ }^{[26]}$.

\section{4 擦拭萃取-表面增强拉曼光谱联用}

擦拭萃取(常被称为擦拭取样)是一种用于固体表 面分析的简便快捷的采样技术，在国土安全、爆炸现 场调查、食品安全、环境检测等公众关注度高的领域 受到广泛关注 ${ }^{[27,28]}$. 擦拭萃取通常获得被分析物的总 量较少，需要高灵敏度的分析技术才能进行下一步分 析，而这正是SERS技术的优势所在，因此结合两者技 术优点的擦拭萃取-SERS联用技术有望在上述公众关 注度高的领域获得广阔的应用.

本课题组开发了带正电荷的铜试剂修饰的银纳米 线膜，用于对典型氧化剂(包括 $\mathrm{ClO}_{4}{ }^{-} 、 \mathrm{ClO}_{3}{ }^{-}$和 $\mathrm{NO}_{3}{ }^{-}$) 的快速现场擦拭提取和灵敏的SERS检测. 通过便携式 拉曼光谱仪仅需几秒钟即可获取可靠的信号，对于 $\mathrm{ClO}_{4}{ }^{-} 、 \mathrm{ClO}_{3}{ }^{-} 、 \mathrm{NO}_{3}{ }^{-} 、 \mathrm{PA}$ 和 2,4-DNP、无机炸药和有 机硝基酮的检出量分别为 $2.0 、 1.7 、 0.1 、 45.8$ 和 $36.6 \mathrm{ng}$. 由于带正电的 $\mathrm{Ag}$ 纳米线膜具有良好的均匀 性、稳定性和可重复使用性，因此还实现了对无机爆 炸性阴离子和有机硝基炸药的SERS定量检测, 表明归 一化拉曼强度与无机爆炸性阴离子浓度之间具有良好 的线性相关性. 该方法可以清楚地检测到模拟无机炸 药(如黑火药、爆竹和火柴)中的典型氧化剂, 包括 $\mathrm{ClO}_{4}{ }^{-} 、 \mathrm{ClO}_{3}{ }^{-}$和 $\mathrm{NO}_{3}{ }^{-}$. 结合便携式拉曼光谱仪，该擦 拭萃取-SERS方法可用于国土安全和爆炸现场调查领 域 ${ }^{[27]}$.

本课题组还开发了带正电荷的铜试剂修饰的银纳 米颗粒，带电的银纳米颗粒胶体可用于便携式拉曼光 谱仪通过静电作用原位检测表面上的无机炸药阴离子 $\mathrm{NO}_{3}{ }^{-}$和 $\mathrm{ClO}_{4}{ }^{-}$. 常见的有机爆炸性苦味酸(PA)也可以通 过相似的相互作用进行检测. 浓缩的DDTC改性的银 纳米颗粒显示出良好的均匀性. SERS强度与炸药阴离
子浓度之间具有良好的线性关系，浓度范围为 $1 \sim$ $500 \mathrm{ng} / \mathrm{cm}^{2}$, 主要由 $\mathrm{NO}_{3}{ }^{-}$和 $\mathrm{ClO}_{4}{ }^{-}$组成的模拟饼干和黑 色粉末在塑料上的检出限可达 $3.521 、 31.6 \mathrm{ng} / \mathrm{cm}^{2}$, 在 织物上可达 $6.47 、 32.2 \mathrm{ng} / \mathrm{cm}^{2}$. 结合便携式拉曼光谱 仪，该原位检测方法可在犯罪现场调查和国土安全方 面对无机阴离子氧化剂、硝基类爆炸物进行痕量分 析 $^{[28]}$.

本课题组发展了一种海绵状的三维柔性铝箔基氧 化锌纳米片阵列基底, 通过在铝箔上生长氧化锌纳米 片阵列并溅射上一层金和银颗粒，在柔性基底上大量 由金和银粒子形成的纳米间隙形成许多热点，可以用 于实际表面增强拉曼光谱检测. 通过SERS性能测试、 红外热成像、祄底老化等测试，验证了该祄底具有良 好的均匀性、热稳定性和长期稳定性. 这种柔性海绵 基底可以通过物理擦拭从各种复杂的样品表面提取分 析物(如杀菌剂福美双). 该基底可以直接用于检测水 果和蔬菜表面的福美双，通过检测 $1382 \mathrm{~cm}^{-1}$ 处的特征 拉曼峰，可实现相对标准偏差为 $6.1 \%$ 的无损循环检测 (图7). 该擦拭萃取基底检出限较低(苹果和番茄表面 的福美双浓度为 $0.2 \mathrm{ng} / \mathrm{cm}^{2}$ )、均匀性好(相对标准偏差 为 $8.9 \%$ )、热稳定性好(相对标准偏差为 $0.9 \%$ ). 该方法 在食品安全、环境监测和公共安全领域有望成为有前 途的快速、现场检测技术 ${ }^{[38]}$.

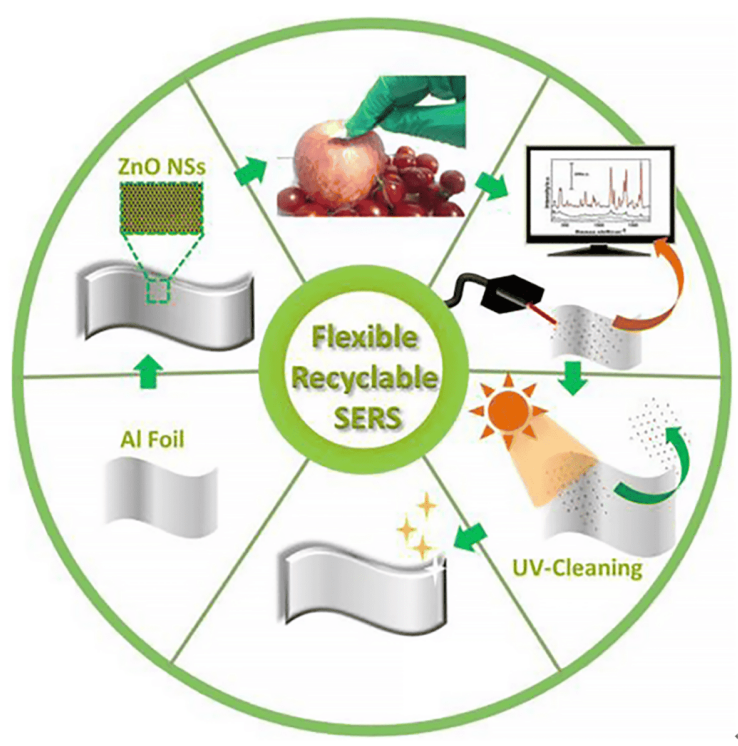

图 7 柔性海绵基底用于擦拭萃取检测福美双的过程示意 图 $^{[38]}$ (网络版彩图)

Figure 7 Process of the in situ swabbing extraction and SERS detection of thiram [38] (color online). 
本课题组通过疏水性丙硫醇修饰银纳米线与便携 式拉曼光谱仪联用，可灵活地原位擦拭提取和检测典 型的多环芳烃. 用乙醇擦拭玩具表面的过程模拟多环 芳烃从玩具到贞儿口的迁移过程，然后使用便携式拉 曼光谱仪在擦拭后几秒钟内获得SERS光谱. 表面覆盖 丙硫醇分子的银纳米线膜表现出对多环芳烃的高亲和 力, 并且被用作定量分析的内标, 苂葱和苯并萠在 $40.45 \sim 28315$ 和 $40.45 \sim 40450 \mathrm{ng} / \mathrm{cm}^{2}$ 的浓度范围内显示 出良好的线性相关性. 此外, 通过使用丙硫醇修饰银纳 米线擦拭萃取SERS检测, 还可以清楚地检测到塑料玩 具和橡胶玩具上的 3 种多环芳烃(苂葱、葸和苯并萠) 的模拟残留. 该方法通过同时进行预处理和分析检测, 只需要较短的总分析时间，在玩具检验领域具有潜在 的应用前景 ${ }^{[39]}$.

\section{5 磁分散固相微萃取-SERS联用技术}

磁分散固相微萃取(MSPME) 是指将萃取相附着 在磁性纳米颗粒表面，没有外加磁场时磁性纳米颗粒 分散到整个样品中萃取被分析物, 然后通过外加磁场 对萃取相进行磁分离, MSPME具有分离速度快、无 有机溶剂、操作简便等特点. 景传勇课题组 ${ }^{[40,56,57]}$ 利 用 $\mathrm{Fe}_{3} \mathrm{O}_{4} @ \mathrm{Ag} 、 \mathrm{Fe}_{3} \mathrm{O}_{4} @ \mathrm{Au} 、 \mathrm{Fe}_{3} \mathrm{O}_{4} @ \mathrm{SiO}_{2}-\mathrm{Au}$ 纳米结构 分别实现了无机砷形态、16种PAHs、食用染料的快 速检测，其对无机砷的检测不仅可以区分三价和五价 砷，还可以实现低至 $10 \mu \mathrm{g} / \mathrm{L}$ 的检测限; 而在PAHs检测 中, 可以根据特征SERS峰区分出 16 种PAHs 且检测限 均可达到 $\mathrm{nM}$ 水平.

徐蔚青课题组 ${ }^{[41]}$ 利用 $\mathrm{Fe}_{3} \mathrm{O}_{4} @ \mathrm{GO} @ \mathrm{Ag}$ 纳米结构建 立了MSPME-SERS联用方法用于检测果皮中的福美 双和噻苯咪唑，最低检测浓度分别为 0.48 和 $40 \mathrm{ng} / \mathrm{cm}^{2}$. 吴玉清课题组 ${ }^{[42,43]}$ 利用 $\mathrm{Fe}_{3} \mathrm{O}_{4} @ \mathrm{GO} @ \mathrm{Ag}$ 和 $\mathrm{Fe}_{3} \mathrm{O}_{4} @$ $\mathrm{SiO}_{2} @ \mathrm{Ag}$ 建立了MSPME-SERS联用方法分别检测蜂 蜜中的氯霉素和保健酒中的枸椽酸西地那非，检测限 分别可以达到 $1.0 \times 10^{-10}$ 和 $1.0 \times 10^{-8} \mathrm{M}$, 单次检测过程 仅耗时 $10 \mathrm{~min}$.

李攻科课题组 ${ }^{[44]}$ 利用埃洛石纳米管(HNTs) 封装 $\mathrm{CoFe}_{2} \mathrm{O}_{4}$ 磁珠, 并在纳米管外壁修饰金纳米颗粒, 以此 建立MSPME-SERS联用方法用于检测化妆品中痕量 4,4-硫代联苯胺以及鱼类饲料和水产样品中的呋喃妥因. 该方法对 4,4-硫代联苯胺和呋喃妥因的线性范围分别 为 $0.054 \sim 21.7 \mathrm{mg} / \mathrm{L}$ 和 $0.05 \sim 1.0 \mathrm{mg} / \mathrm{L}$, 检出限分别为
0.026 和 $0.014 \mathrm{mg} / \mathrm{L}$ ，加标回收率分别为 $71.6 \%$ 103.6\% 和 $81.9 \% \sim 116.3 \%, \mathrm{HNTs}$ 封装 $\mathrm{CoFe}_{2} \mathrm{O}_{4}$ 磁珠可以增强氨 基功能化和氮杂环分析物的吸附, 并降低粒子的聚集.

\section{6 薄层微萃取-SERS联用技术}

薄层微萃取(TFME) 是一种将薄膜置于水样(浸渍) 或固体或液体样(顶空)之上以提取被分析物的技术, 其具有比表面积大、提取层薄、提取层相对均匀、提 取效率高、提取平衡时间短、灵敏度高等特点, TFME的薄层平板结构与常见的SERS基底具有高度 吻合性，可以充分利用现有的SERS基底制备方法. 王 翊如课题组 ${ }^{[45]}$ 利用海胆状 $\mathrm{ZnO}$ 材料作为顶空薄层微萃 取相萃取红酒中的 $\mathrm{SO}_{2}$, 然后滴加金纳米颗粒作为 SERS基底, 该方法可成功地应用于实际样品酒中的 $\mathrm{SO}_{2}$ 的检测, 对其检测的线性范围为 $1 \sim 200 \mu \mathrm{g} / \mathrm{mL}$, 检 出限为 $0.1 \mu \mathrm{g} / \mathrm{mL}$, 与传统的 $\mathrm{SO}_{2}$ 测定方法相比, 该方法 具有操作简单、快速、灵敏度高等明显优势. 该方法 还可用于中草药中亚硫酸盐的测定, 检出限为 $6 \mathrm{mg} / \mathrm{kg}$, 与传统蒸馏-滴定方法耗时 $1 \mathrm{~h}$ 以上相比, 该 方法总分析时间只需要 $10 \mathrm{~min}^{[46]}$. 王靖如课题组 ${ }^{[47]}$ 还 利用单壁碳纳米管-氧化物试纸条结合金纳米颗粒检 测湖水中的西草净农药残留, 可以达到 $2 \mu \mathrm{g} / \mathrm{L}$ 的检测 限和 $70 \% \sim 80 \%$ 的回收率. 此外, 该课题组 ${ }^{[4]}$ 利用商业 硅胶板和金纳米颗粒检测碳酸饮料中的苯甲酸钠, 该 方法的检出限为 $3.6 \mu \mathrm{g} / \mathrm{m} \mathrm{L}$, 加标回收率为 $85.0 \% \sim 103.0 \%$.

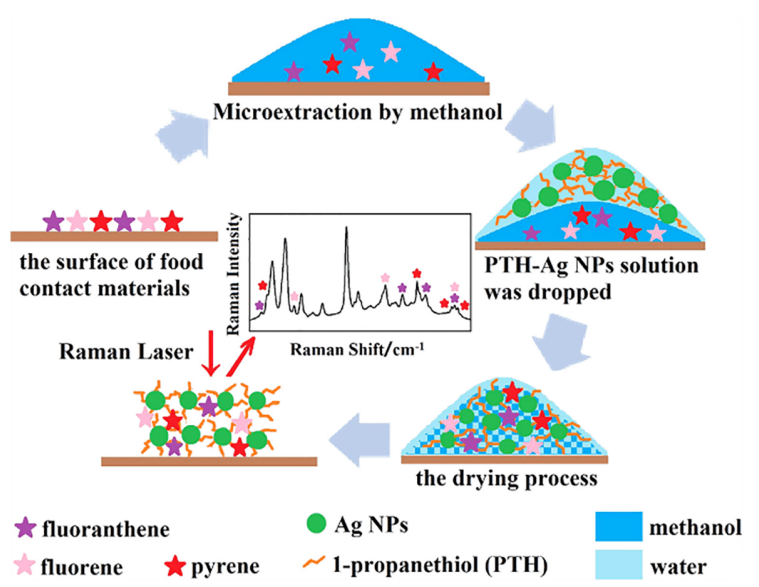

图 8 原位微萃取-SERS联用方法用于检测食品接触材料表 面的多环芳烃过程示意图 ${ }^{[49]}$ (网络版彩图)

Figure 8 Process of the in-situ microextraction and SERS detection of PAHs [49] (color online). 


\section{7 其他液相微萃取-表面增强拉曼光谱联用}

本课题组开发了一种结合表面增强拉曼光谱和原 位微萃取技术的联用技术，用于食品接触材料上多环 芳烃的现场篎选(图8). 分别使用甲醇和丙硫醇修饰的 银纳米颗粒进行原位微萃取和多环芳烃检测, 选择丙 硫醇的 $\mathrm{C}-\mathrm{C}$ 键在 $1030 \mathrm{~cm}^{-1}$ 处的振动作为内标峰, 通过 便携式拉曼光谱仪就可以获得SERS光谱. 该SERS方 法具有较高的均一性, RSD为 $2.96 \%$. 归一化的SERS 强度与苂葱浓度的关系图在0.789 158 ng/ $\mathrm{cm}^{2}$ 的浓度 范围内显示出线性关系 $\left(R^{2}=0.98\right)$. 检出限可达 $0.27 \mathrm{ng} / \mathrm{cm}^{2}$. 这种原位微萃取-SERS联用方法证明了 其能够快速篮选污染的食品接触材料上的多环芳烃, 而无需进行复杂的样品预处理 ${ }^{[49]}$.

本课题组采用分散的液-液微萃取(DLLME)技术, 研制了一种便于高通量的多环芳烃SERS分析试剂盒. 该便携式试剂盒包括三个密封的试剂管(标记为 $\mathrm{T} 1$ 、

$\mathrm{T} 2$ 和 T3)、自制的孔板和便携式拉曼光谱仪. 试剂管 T1包含分散剂溶剂和萃取溶剂的混合物，其中涉及 DLLME工艺的 $2 \mathrm{~min}$ 样品预处理. 将 T1管中的溶剂快 速注入含有多环芳烃的样品中，立即形成一种浑浊的 溶液，该溶液由完全分散在水相中的萃取溶剂细滴组 成, 富集因子为29.6. T2和 T3 分别含有甲醇和1-丙硫醇 修饰的银纳米粒子. 在自制的高通量微反应器上, 利用 $\mathrm{T} 3$ 管中的液体增强被测物的拉曼信号，可得到萠浓度 与相对拉曼峰强度的线性关系 $\left(R^{2}=0.993\right)$, 萠的检出限 为 $0.50 \mu \mathrm{g} / \mathrm{L}$. 该方法用于 12 个同浓度样品高通量分析 时，其相对标准偏差为 $4.8 \%$. 对于水样中多环芳烃异 构体及其混合物，该方法可以通过典型拉曼峰和主成 分分析(PCA)进行鉴别. 该DLLME-SERS方法具有现 场高通量分析多种多环芳烃的潜力 ${ }^{[50]}$.

\section{4 总结与展望}

基于萃取和表面增强拉曼光谱技术分别作为样品
前处理和分析测定方法的Ex-SERS技术，可以融合 SERS与萃取技术各自的特点, 克服单一技术中存在的 问题. 相比单独的SERS技术，Ex-SERS联用技术的优 势体现在: (1) 扩大了SERS的应用范围, 使之能用于分 析与SERS基底无强相互作用的化合物; (2) 隔绝了样 品基质与SERS基底的直接接触, 将基质中的大部分干 扰物排除到SERS基底有效增强区域以外，从而有效避 免其对SERS分析的影响; (3) 被分析物与SERS基底无 直接接触, 避免导致峰位置发生较大偏移的化学增强 过程发生, 有助于减少峰位置偏移带来的定性困难; （4）萃取相赋予SERS基底上不同区域对被分析物相同 的吸附性能，减少了由于激光聚焦位置不同带来的信 号差异; (5) 被分析物总是与萃取相处于相同的SERS 增强环境中, 可以利用萃取相作为内标来实现定量分 析. 相比单独的萃取技术, Ex-SERS联用技术的优势体 现在: (1) 萃取相中的被分析物无需进一步的转移和处 理, 减少了转移和处理过程中的被分析物损失; (2) 前 处理和分析测定过程的一体化可极大地缩短样品分析 总时间, 提高样品分析效率.

同样，Ex-SERS联用技术也存在诸多不足：首先 Ex-SERS过程中萃取得到的往往不是单一的被分析 物, 而是一类物理化学性质相似的物质, 这势必加大样 品信号处理过程中的难题; 其次, 现有方法基本都是基 于实验室模拟样品开发的，其在复杂和多样化基质的 实际样品中的效果有待进一步考察; 最后Ex-SERS联 用技术的应用跨越多个学科范畴, 需要联合多学科研 制针对具体使用场景的Ex-SERS仪器和建立相关分析 方法.

虽然可以预见Ex-SERS联用技术在实际样品的现 场分析中大有可为, 但现有的研究还不足以使 ExSERS联用技术走出实验室. 因此，我们期待在国内外 课题组的共同努力下，基于Ex-SERS联用技术的分析 方法和仪器能走出实验室, 应用到人类生产和生活的 各个方面.

致谢感谢占金华课题组在过去十余年来在Ex-SERS领域努力工作的成员：段俊玲博士、来永超博士、王玮博士、 姜小红博士、崔精诚博士、史玉娥博士、张旻博士、杨衡博士、边玮玮博士、孟艳静、袁敬鹏、荆丽晓、陈娟、李 博、刘翠翠、朱莎、孙雷、肖兰兰、于晓飞、王颖. 特别感谢本课题组的合作者姜炜教授、张晓丽教授, 祝山东大学 120 周年生日快乐. 


\section{参考文献}

1 Zong C, Xu M, Xu LJ, Wei T, Ma X, Zheng XS, Hu R, Ren B. Chem Rev, 2018, 118: 4946-4980

2 Zrimsek AB, Chiang N, Mattei M, Zaleski S, McAnally MO, Chapman CT, Henry AI, Schatz GC, Van Duyne RP. Chem Rev, 2017, 117: 75837613

3 Stiles PL, Dieringer JA, Shah NC, van Duyne RP. Annu Rev Anal Chem, 2008, 1: 601-626

4 de Albuquerque CDL, Sobral-Filho RG, Poppi RJ, Brolo AG. Anal Chem, 2018, 90: 1248-1254

5 Dong R, Li S, Lin D, Chen H, Yang L. Sci Sin-Chim, 2021, 51: 294-309 (in Chinese) [董荣录, 李绍飞, 林东岳, 陈慧, 杨良保. 中国科学: 化学, 2021, 51: 294-309]

6 Panneerselvam R, Liu GK, Wang YH, Liu JY, Ding SY, Li JF, Wu DY, Tian ZQ. Chem Commun, 2018, 54: 10-25

7 Sharma B, Frontiera RR, Henry AI, Ringe E, Van Duyne RP. Mater Today, 2012, 15: 16-25

8 Lin J, Liang L, Ling X, Zhang S, Mao N, Zhang N, Sumpter BG, Meunier V, Tong L, Zhang J. J Am Chem Soc, 2015, 137: 15511-15517

9 Zheng Z, Cong S, Gong W, Xuan J, Li G, Lu W, Geng F, Zhao Z. Nat Commun, 2017, 8: 1993

10 Nie S, Emory SR. Science, 1997, 275: 1102-1106

11 Craig AP, Franca AS, Irudayaraj J. Annu Rev Food Sci Technol, 2013, 4: 369-380

12 Sun F, Hung HC, Sinclair A, Zhang P, Bai T, Galvan DD, Jain P, Li B, Jiang S, Yu Q. Nat Commun, 2016, 7: 13437

13 de Nijs B, Carnegie C, Szabó I, Grys DB, Chikkaraddy R, Kamp M, Barrow SJ, Readman CA, Kleemann ME, Scherman OA, Rosta E, Baumberg JJ. ACS Sens, 2019, 4: 2988-2996

14 Shen W, Lin X, Jiang C, Li C, Lin H, Huang J, Wang S, Liu G, Yan X, Zhong Q, Ren B. Angew Chem Int Ed, 2015, 54: 7308-7312

15 Tang S, Zhang H, Lee HK. Anal Chem, 2016, 88: 228-249

16 Musteata FM, Pawliszyn J. TrAC Trends Anal Chem, 2007, 26: 36-45

17 Chen XH. Solid Phase Extraction Technology and Its Application. Beijing: Science Press, 2019 (in Chinese) [陈小华. 固相萃取技术与应用. 北 京: 科学出版社, 2019]

18 Ouyang GF, Pawliszyn J. Solid Phase Microextraction: Principle and Application. Beijing: Chemical Industry Press, 2012 (in Chinese) [欧阳刚 锋, Pawliszyn J. 固相微萃取: 原理与应用. 北京: 化学工业出版社, 2012]

19 Kennedy BJ, Spaeth S, Dickey M, Carron KT. J Phys Chem B, 1999, 103: 3640-3646

20 Lai YC. Surface Enhanced Raman Spectroscopy for Rapid Analysis of Environmental Organic Pollutants. Dissertation for Doctoral Degree. Jinan: Shandong University, 2013 (in Chinese) [来永超. 面向环境有机污染物快速分析的表面增强拉曼光谱方法. 博士学位论文. 济南: 山东 大学, 2013]

21 Kang T, Hong S, Choi Y, Lee LP. Small, 2010, 6: 2649-2652

Lai Y, Pan W, Zhang D, Zhan J. Nanoscale, 2011, 3: 2134-2137

Liu Z, Wang L, Bian W, Zhang M, Zhan J. RSC Adv, 2017, 7: 3117-3124

Bian W, Liu Z, Lian G, Wang L, Wang Q, Zhan J. Anal Chim Acta, 2017, 994: 56-64

i J, Liu H, Chen J, Zeng J, Huang J, Gao L, Wang Y, Chen X. J Chromatogr A, 2012, 1246: 22-27

Shi Y, Wang W, Zhan J. Nano Res, 2016, 9: 2487-2497

Chen J, Shi Y, Zhang M, Zhan J. RSC Adv, 2016, 6: 51823-51829

Jiang X, Lai Y, Wang W, Jiang W, Zhan J. Talanta, 2013, 116: 14-17

Lai Y, Cui J, Jiang X, Zhu S, Zhan J. Analyst, 2013, 138: 2598-2603

Yu X, Chang Y, Natarajan V, Zhang X, Zhan J. Anal Methods, 2018, 10: 1353-1361

Feng J, Hu Y, Grant E, Lu X. Food Chem, 2018, 239: 816-822

Hu R, Tang R, Xu J, Lu F. Anal Chim Acta, 2018, 1034: 176-183

Wang C, Zhang Z, He L. J Raman Spectrosc, 2019, 50: 6-14

Bian W, Zhu S, Qi M, Xiao L, Liu Z, Zhan J. Anal Methods, 2017, 9: 459-464 
Qiu L, Liu Q, Zeng X, Liu Q, Hou X, Tian Y, Wu L. Talanta, 2018, 187: 13-18

Cui J, Chen S, Ma X, Shao H, Zhan J. Microchim Acta, 2019, 186: 19

Wang Y, Yu X, Chang Y, Gao C, Chen J, Zhang X, Zhan J. Microchim Acta, 2019, 186: 458

Xiao L, Zhang M, Liu Z, Bian W, Zhang X, Zhan J. Anal Methods, 2017, 9: 1816-1824

Du J, Cui J, Jing C. Chem Commun, 2014, 50: 347-349

Liu Z, Wang Y, Deng R, Yang L, Yu S, Xu S, Xu W. ACS Appl Mater Interfaces, 2016, 8: 14160-14168

Yu S, Liu Z, Li H, Zhang J, Yuan X, Jia X, Wu Y. Analyst, 2018, 143: 883-890

Yu S, Liu Z, Wang W, Jin L, Xu W, Wu Y. Talanta, 2018, 178: 498-506

Zhang H, Lai H, Wu X, Li G, Hu Y. Anal Chem, 2020, 92: 4607-4613

Deng Z, Chen X, Wang Y, Fang E, Zhang Z, Chen X. Anal Chem, 2015, 87: 633-640

Dong J, Cai L, Wang S, Wang Y, Chen X. Anal Lett, 2018, 52: 1236-1246

Deng Z, Liu H, Wang Y, Chen X. Anal Methods, 2015, 7: 2190-2195

Cai L, Dong J, Wang Y, Chen X. Talanta, 2018, 178: 268-273

Zhang M, Zhang X, Shi Y, Liu Z, Zhan J. Talanta, 2016, 158: 322-329

Zhang M, Zhang X, Qu B, Zhan J. Talanta, 2017, 175: 495-500

Song Y, Huang HC, Lu W, Li N, Su J, Cheng SB, Lai Y, Chen J, Zhan J. Food Chem, 2021, 344: 128570

Lai Y, Wang C, Shao H. ACS Appl Nano Mater, 2020, 3: 1800-1807

Zhu S, Zhang X, Cui J, Shi Y, Jiang X, Liu Z, Zhan J. Analyst, 2015, 140: 2815-2822

Liu C, Zhang X, Li L, Cui J, Shi Y, Wang L, Zhan J. Analyst, 2015, 140: 4668-4675

Jiang X, Lai Y, Yang M, Yang H, Jiang W, Zhan J. Analyst, 2012, 137: 3995-4000

Sun Z, Du J, Yan L, Chen S, Yang Z, Jing C. ACS Appl Mater Interfaces, 2016, 8: 3056-3062

Du J, Xu J, Sun Z, Jing C. Anal Chim Acta, 2016, 915: 81-89 


\title{
Development of extraction-surface enhanced Raman spectroscopy and its application in detection of hazardous materials
}

\author{
Yongchao Lai ${ }^{1}$, Jing Chen ${ }^{2}$, Jinhua Zhan ${ }^{2 *}$ \\ ${ }^{1}$ Science and Technology Innovation Center, Shandong First Medical University \& Shandong Academy of Medical Sciences, Jinan 250062, China \\ ${ }^{2}$ Key Laboratory for Colloid and Interface Chemistry of Ministry of Education, School of Chemistry and Chemical Engineering, Shandong University, \\ Jinan 250100, China \\ *Corresponding author (email: jhzhan@sdu.edu.cn)
}

\begin{abstract}
Surface enhanced Raman spectroscopy (SERS), which is known for its high sensitivity and molecular characteristics of the fingerprint spectrum, has been used in many fields. SERS might be interfered by the sample matrix and the impurity especially when trace analyte is being analyzed, which limits its application in actual samples analysis. Therefore, extraction-surface enhanced Raman spectroscopy (Ex-SERS) hyphenated technique is proposed to solve this problem. Several extraction technologies including solid phase extraction, solid phase microextraction, micro magnetic dispersive solid-phase microextraction, thin layer chromatography extraction, liquid-liquid dispersion microextraction have been used to construct Ex-SERS hyphenated technique. A variety of Ex-SERS methods were developed for rapid in-situ separation and detection of trace analytes from complex sample, which could promote the application of SERS technology in actual sample analysis.
\end{abstract}

Keywords: surface enhanced Raman spectroscopy, extraction, sample pretreatment, hazardous materials, hyphenated technique

doi: $10.1360 /$ SSC-2021-0030 